\title{
Investigation on Digital Media Image Processing Algorithm Based on Asynchronous and Inertia Adaptive Particle Swarm Optimization
}

\author{
Wenchao Zhang, Caigen Zhou and Xinxin Bao \\ Department of Art and Design, Changzhou College of Information Technology, \\ Changzhou, Jiangsu, China \\ Wenchao Zhang E-mail:576710599@qq.com \\ Caigen Zhou E-mail:411510506@qq.com \\ Xinxin Bao E-mail: 2050319152@qq.com
}

\begin{abstract}
With the continuous development of computer science and technology, image processing and analysis gradually form the scientific system. Although history of image processing is not long, it attracts many researchers study on it. Digital media image widely exists in many fields, such as education, video, advertisement, and so on. Process digital media image is an important part of image processing. When analyze the digital media image, we want to extract the image part we care from the original image and then method for image segmentation is quite important. That is to say that the image segmentation will divide the image into a number of regions with specific and unique nature. How to keep the original characteristics of the digital media image is quite important in the image segmentation. In this paper, we propose a new algorithm for digital media image segmentation, and it is also can be used in the image processing. The algorithm is based on asynchronous particle swarm optimization algorithm to obtain the adaptive threshold; take the inertia factor into the algorithm, the optimal threshold has been acquired for the image segmentation. Compared with other particle swarm optimization algorithm, the algorithm has the advantages of stable, easy to converge to the optimal solution, and high segmentation speed.
\end{abstract}

Keywords: digital media, image segmentation, PSO, asynchronous, adaptive factor

\section{Introduction}

The digital media image processing technology is an interdisciplinary field. With the continuous development of computer science and technology, image processing and analysis gradually formed the scientific system, and so do the digital media image. Digital media image is widely used in the society, such as education, advertisement, video, film, and so on. Processing algorithm for the digital media image is also quite important for us to get vivid image. So, many treatment methods have been emerged. Although its history is not long, it attracts concern of various researchers. Firstly, the vision is the most important means of human perception and the digital media image is the basis of vision. Therefore, the digital media image has become an effective tool in the research for the scholars study on visual perception psychology, physiology, computer science and other fields. Secondly, digital media image processing has great demand in the, remote sensing, meteorological and other large applications.

In digital media image analysis, target part is often required to extract from the image. Image segmentation can be described as that a particular region is separated and extracted from the other parts of the image. That is to say that the digital media image segmentation will divide the image into a number of regions with specific and unique natures, and 
propose the technique and process for the target of interest. It is the fundamental step from image processing to image analysis. The existing image segmentation methods can be mainly classified into the following categories: (1) Based on the threshold segmentation method; (2) Segmentation method based on region; (3) Segmentation method based on edge; and (4) Specific segmentation method based on the theory. Since 1998, researchers continue to improve the original method of image segmentation and many new theories of other disciplines and many new methods have been proposed. The extract target can be used for semantic image recognition, image search, etc.

In this paper, we propose a new algorithm for digital media image segmentation, and it can also be used in the image processing. The algorithm is based on asynchronous particle swarm optimization algorithm to obtain the adaptive threshold and take the inertia factor into the algorithm to get the optimal threshold for the digital media image segmentation. The main contribution of the paper is to propose a comprehensive method for the digital media image segmentation. And the remainder of the paper is shown as the following: image segmentation algorithms are listed in Section 2. The new algorithm for image segmentation is shown in Section 3. Experimental Analysis is described in Section 4. And the conclusion is shown in Section 5.

\section{Several Image Segmentation Algorithms}

\subsection{Segmentation Method based on Region}

The region based segmentation method is a segmentation algorithm with region directly searches. Specific algorithms include region growing and region separation and merging. The region based separation and extraction method has two basic forms: One is the regional growth, which is starting from a single pixel and gradually merged to form the segmented regions; the other is from the whole region and gradually cut to segmentation region needed. In actual condition, two basic forms of the algorithms are usually used comprehensively. This method has a good effect in the application of complex scene for complex objects or for some natural scene segmentation.

\subsection{Segmentation Method based on Edge}

Edge based segmentation method solves problems through edge detection in different regions. Gray value on the edge of different regions is often relatively large, and it is one of the main assumptions of the edge detection method can be realized. Its basic idea is to detect the image edge points, which are then connected to a profile according to a certain strategy. Its difficulty lies in the contradiction between the noise immunity on the edge detection and the detection precision. If improve the detection precision, unreasonable profile will be generated by the noise. If improve noise immunity will lead to detection missing and position deviation.

\subsection{Image Segmentation Method Combining Edge and Region}

Edge detection can obtain the local variation intensity of gray value, while regional segmentation can detect features similarity and homogeneity. Combining edge and region segmentation has both advantages of the two methods. Through the edge point limit, regions over segmentation will be avoided, and region segmentation can be used to add the missing edge to make the contour more complete

\subsection{Segmentation Method based on the Specific Theory}

In recent years, many new theories and new methods have been developed, some specific theories, methods and tools have also been proposed for the image segmentation. In fact, image segmentation technology has no general theory, once there are new mathematical tools or methods proposed it will be tried to use in image segmentation. Then, many special algorithms have been put forward. 
(1) Image segmentation method based on mathematical morphology [1-4]

Mathematical morphology takes morphological characteristics of the image as the study objects. Elements with a certain form are used to describe the relation among elements and parts to achieve purpose of image analysis and identification. Mathematical morphology used in image segmentation can be combined with edge based methods and region based methods. Most typical mathematical morphology for the image segmentation is watershed based method. The classical watershed method mainly consists of two steps: "sort" and" flooded". In the sort step, it mainly completes the calculation of frequency distribution of gray level of the image. Gray level will be ordered according to the calculation results, and then each pixel in the image will be removed to the memory array corresponds with the gray level. In the process of flooded, recursive arithmetic are used to realize expanding of the water basin and finally complete the image segmentation. Plenty of improved algorithms have also been developed based on the watershed method.

(2) Image segmentation based on Fuzzy Theory [5-8]

Fuzzy techniques can be used for image segmentation, and guiding ideology and starting point is that the result of image segmentation should be a fuzzy subset defined in pixel space rather than children subset. This is because, in many cases, especially with the window size of $3 \times 3$ or $5 \times 5$, homogeneous characteristics may not be drastic changes in the border region, and it is difficult to determine whether a pixel should belong to a region. At this time, for each pixel and each region, membership value, which represents a pixel belonging to the region, is assigned to each pixel. When take the nature of the region into consideration, membership degree can be used to obtain an accurate estimation of the regional property. The basic steps of application of fuzzy techniques for image segmentation is that: 1) Image and feature representation should be expressed into the corresponding fuzzy sets and fuzzy concept; 2) After processing by fuzzy technology, get the fuzzy image segmentation; 3) Image segmentation results can be obtained after diversification.

(3) Other specific theories

Other specific theories includes image segmentation method based on Neural Network [9-17], Image segmentation method based on support vector machine [18-20], image segmentation method based on graph theory [21-22], image segmentation method based on immune algorithm [23], image segmentation method based on granular computing theory[24-27], and so on.

\subsection{Two Dimensional Entropy Methods}

The entropy image segmentation method can be classified into one-dimensional and two-dimensional methods. One-dimensional entropy image segmentation method uses the gray information of the image. When the signal to noise ratio (SNR) is low, the image segmentation will have a bad effect. Then one-dimensional entropy image segmentation method is extended into a two-dimensional entropy method.

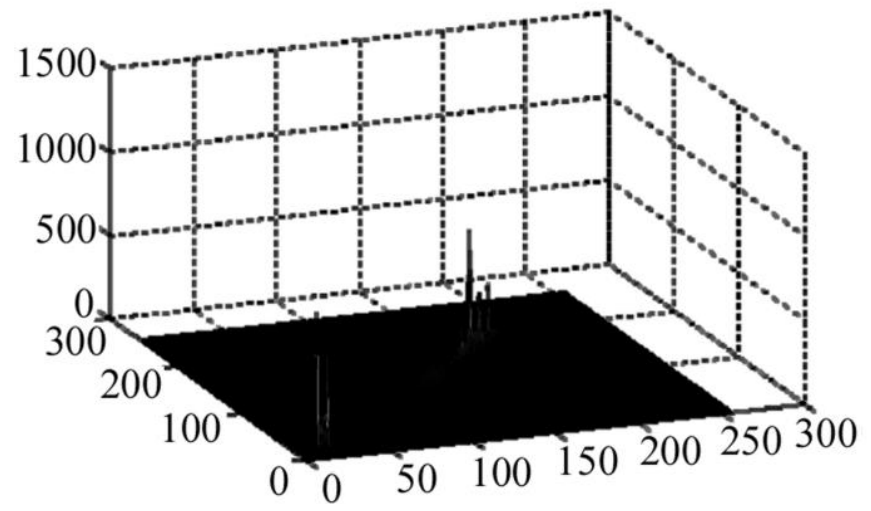

Figure 1. Gray-mean Value of Local Gray 
This method uses the mean value of gray value and local gray value of image pixels to process the image. The design idea of the two dimension entropy method is the information correlation between pixel and neighborhood is described as two dimension entropy. To measure the related information of neighborhood, a simple and effective method is to describe it with the mean value of pixels in a certain size of the template. Then, when the spatial correlation information amount is maximum, that is the best threshold.

Take the image gray as horizontal axis and local gray mean as the ordinate axis to establish a scatter diagram, as shown in Figure 1. Diagonal points are corresponding to the internal object or background while the diagonal leaving points are corresponding to the boundary of the point.

The specific steps of 2-D entropy method are as follows:

(1) Calculation of the joint intensity for each pixel $p(i, j)$ :

$$
p(i, j)=\frac{N_{i j}}{N_{\text {image }}}, \quad i=0,1,2, \ldots, 255 ; \quad j=0,1,2, \ldots, 255
$$

Where, $N(i, j)$ is the number of pixels with gray value of $i$ and with mean gray value of $j ; N_{\text {image }}$ is the total number of pixels of the image.

(2) Set the initial threshold as $T h_{s t}=T h_{s t}(0)$, and the image would be divided into two parts of $C_{1}$ and $C_{2}$;

(3) Calculate mean relative two dimension entropy of the two parts:

$$
\begin{gathered}
E_{1}=-\sum_{i=1}^{s} \sum_{j=1}^{t} \frac{p_{i j}}{p_{s t}} \ln \frac{p_{i j}}{p_{s t}} \\
E_{2}=-\sum_{i=s+1}^{255} \sum_{j=t+1}^{255} \frac{p_{i j}}{1-p_{s t}} \ln \frac{p_{i j}}{1-p_{s t}}
\end{gathered}
$$

Where, $E_{1}=\sum_{i=1}^{s} \sum_{j=1}^{t} p_{i j}$;

(4) Select the optimal threshold $T h_{s t}=T h_{s t}^{*}$, and the image will meet the demand of the equation (4) described after been divided into $C_{1}$ and $C_{2}$;

$$
\left[E_{1}+E_{2}\right]_{T h_{s t}=T h_{s t}^{*}}=\max \left\{E_{1}+E_{2}\right\}
$$

\section{The New Algorithm}

\subsection{PSO}

PSO simulates predation behavior of birds. From the inspiration from this process, PSO is widely used to solve the optimization problem. In this paper, two definitions are firstly given:

(1) Particle: Each solution of the optimization problem is regarded as a bird in the space with $D$ dimension, which is called the "particle". $X$ is a space particle with the dimension of $D$.

(2) Particle Swarm: Population $n$ is composed of $N$ particles (repeated particles are allowed), and the population is referred as the particle swarm. $N$ is the size of the particle swarm.

In PSO, particle flies in the search space at a speed, and it adjusts their flight according to the flight experience of itself and peers. This adjustment is expressed as the particle updating their own information through two extreme values. One of the extreme values is the best position the particle experienced itself, which is marked as pbest. The other extreme value is the best position of the whole population currently experienced, which is 
called the global extreme value pgbest. All the particles have fitness values determined by optimized function to evaluate the quality of current positions of particles. In addition, part of the particle population can be taken as the neighbor, and the global extremum can be regarded as the optimal local extreme values in the neighbor particles. During the flight of the particles, through mutual exchange between individuals and populations and selfupdating, optimal position or its adjacent regions will be eventually arrived. In each iteration (flight), the particles follow global optimization:

$$
\begin{gathered}
V_{k}=V_{k}+c_{1} \times \operatorname{rand}() \times\left(\text { pbest }_{k}-x_{k}\right)+c_{2} \times \operatorname{rand}() \times\left(\text { gbest }_{k}-x_{k}\right) \\
x_{k}=x_{k}+V_{k}
\end{gathered}
$$

Where, $V_{k}$ is the velocity of the particle, and $x_{k}$ is the current optimal position. pbest is the optimal position of the particle finds, and pgbest is the optimal position of the whole swarm finds.

The above is the expression of PSO, and this expression has been modified as the following:

$$
V_{k}=\omega \times V_{k}+c_{1} \times \operatorname{rand}() \times\left(\text { pbest }_{k}-x_{k}\right)+c_{2} \times \operatorname{rand}() \times\left(\text { gbest }_{k}-x_{k}\right)
$$

Where, $\omega$ is the inertia factor and balances the global and local search. It can be a positive constant or even a positive linear or nonlinear function of time. The flowchart of PSO is shows in Figure 1.

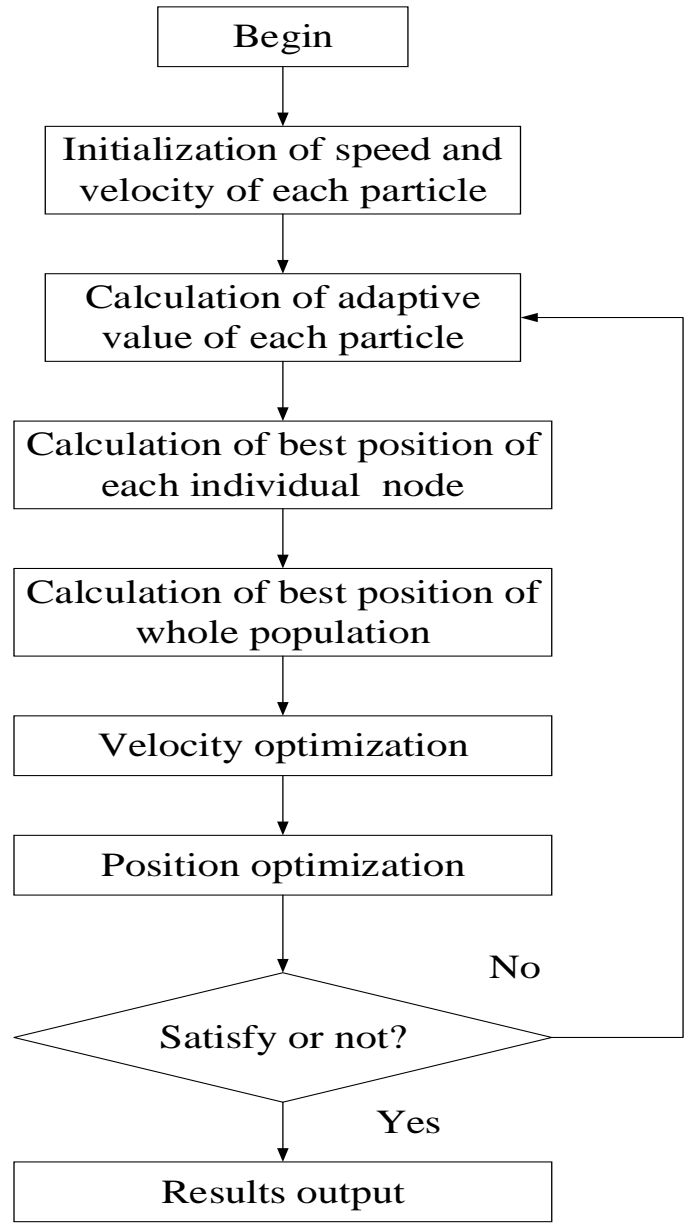

Figure 1. Flowchart of PSO Method 


\subsection{Asynchronous PSO}

Standard PSO algorithm is divided into two stages. One stage is to determine the fitness value of each particle, individual and global maxima and minima. The other stage is to determine the speed and update each particle, and standard PSO algorithm usually contains two groups. An individual is updated by the individual and global maxima and minima calculated by information gained from former a group. Generating new individual is added to the current group.

However, asynchronous PSO algorithm has only one stage: fitness value of each particle, individual extreme, global extremes, speed and position are continuously updated. Each particle of the group is updated one by one. Therefore, in each iteration, the current update will take into account the new information generated by all the previous updated particles, And in an asynchronous particle swarm optimization algorithm, all particles will be sorted according to fitness value of each particle before the start of each iteration, with optimal fitness value at the front and poor fitness valued at the back. So that when the update begins, the better the fitness value of particles is, the sooner the particle is updated, which will provide more useful information for the update later particles. Asynchronous particle swarm algorithm requires only one population, each updating a particle; the former particle will be updated automatically replaced by the updated particle.

\subsection{Optimal Threshold Image Segmentation based on Asynchronous PSO}

(1) Clustering optimal threshold choice

The clustering pattern recognition ideas are used in digital media image segmentation. The basis is as follows: The purpose is to keep maximum similarity of class within the model to the sample (i.e., pixels) and keep the maximum distance between the various types. The optimal threshold for digital media image segmentation is gained from Diego expression law.

The steps are as follows:

1) Random given threshold to target digital media image divides an image into target c1 and c2 background. The two probabilities are:

$$
\left\{\begin{array}{l}
p_{1}=n c_{1} / N_{\text {image }} \\
p_{1}=n c_{1} / N_{\text {image }}
\end{array}\right.
$$

$n c_{i}$ is the number of pixels of the class $c_{i},(i=1,2) . N_{\text {image }}$ is the total pixels number in the image. Two types of centers gray level average value $u_{i}$ and variance $\sigma_{i}^{2}$ are as follows:

$$
\begin{aligned}
u_{i} & =\frac{1}{n c_{i}} \sum_{(x, y) \in c_{i}} f(x, y) \\
\sigma_{i}^{2} & =\sum_{(x, y) \in c_{i}}\left(f(x, y)-u_{i}\right)^{2}
\end{aligned}
$$

$$
\text { Mark } h\left(t_{1}\right)=p_{1} \cdot \sigma_{1}^{2}+p_{2} \cdot \sigma_{2}^{2}
$$

2) Classify each pixel in the image. The method is as follows:

$$
(x, y) \in\left\{\begin{array}{l}
c_{1}, \quad \text { if }\left|f(x, y)-u_{1}\right| \leq\left|f(x, y)-u_{2}\right| \\
c_{2}, \text { else }
\end{array}\right.
$$

3) After the classification, the mean center gray value of all the pixels in the target and background should be calculated: 


$$
h\left(t_{2}\right)=\sum_{i=1}^{2} p_{i}^{\text {new }} \sigma_{i}^{2(\text { new })}
$$

The threshold should be meeting the requirements: If $h\left(t_{2}\right)<h\left(t_{1}\right)$, pixels should be reclassified, or else, $t_{1}$ will be selected as the threshold.

Then, determination of optimal threshold should be satisfy the demand as the following:

$$
t^{*}=\underset{0 \leq t \leq l-1}{\arg \min } h(t)
$$

(2) Improved particle swarm optimization algorithm for digital media image segmentation

Image segmentation problem can be seen as a particle swarm optimization problem by using optimization capability of particle swarm. Through mutual learning between particles, the best position will be found and the optimal threshold for digital media image segmentation will be determined.

Since the gray level of the pixel is 256 , the initial position and velocity of the particles in the experiment, and each iteration of the position and velocity of the particles is limited in the $0-255$.

Algorithm steps are shown in Figure 2.

Set the parameters:

particles size: $n_{P}$

initial velocity and position

Maximum iteration: $\mathrm{n}_{\max }$

Counter: $\mathrm{n}_{\mathrm{c}}$

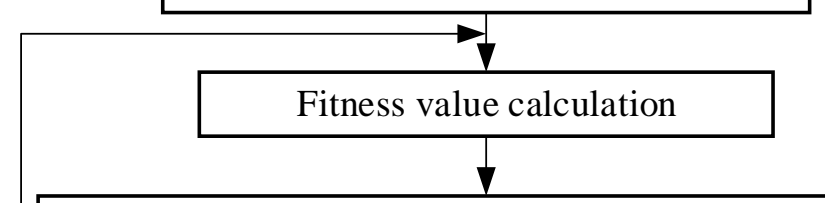

Find the optimal global extreme value $p$ gbest according to the current fitness value of each particle

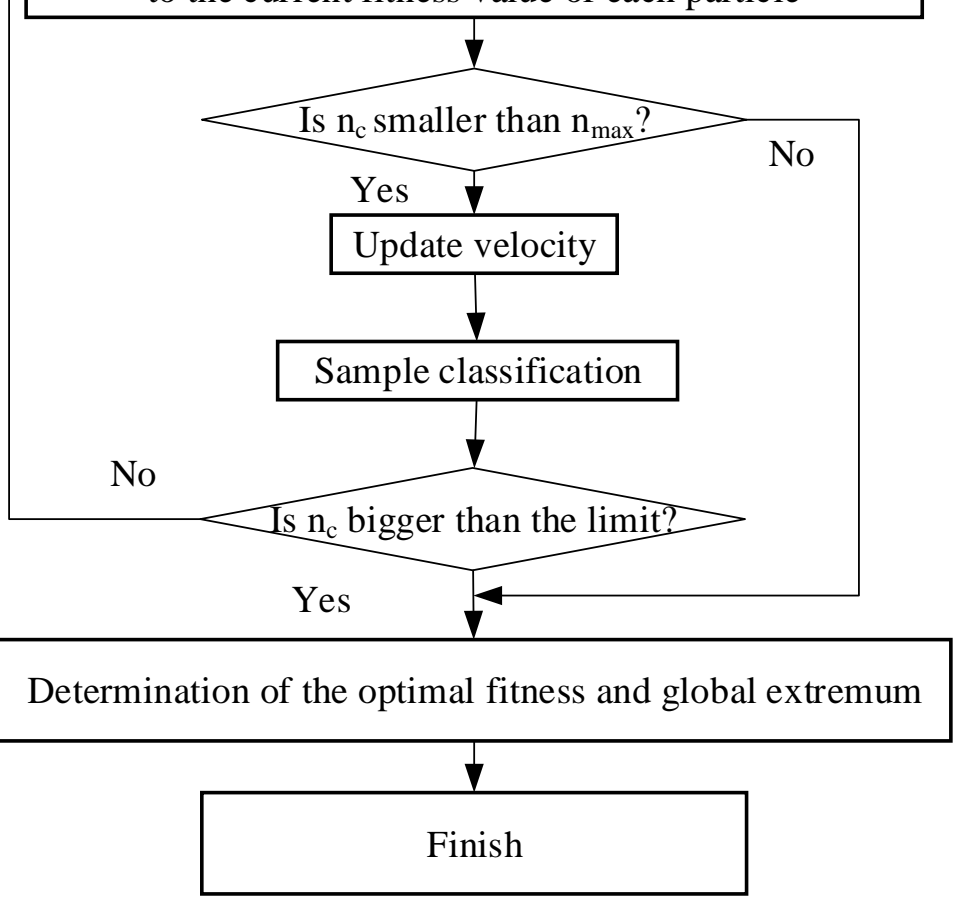

Figure 2. Flow Chart of Optimal Threshold Image Segmentation 


\subsection{Inertia Adaptive PSO Algorithm}

If most particles in the swarm stop the search before successive finding the optimal values, it will appear the phenomenon of premature convergence. When the inertia weight is smaller or fixed, it will be easy to appear. When $v_{i j}(t)$ and $\left|p_{i j}(t)-x_{i j}(t)\right|$ and $\left|p_{g j}(t)-x_{i j}(t)\right|$ are small, it means the search ability of corresponding particles is lost. This situation often occurs when the particle itself is the optimal global position and it means the particles in later iterations lose the diversity. In order to solve this problem, $\omega$ is often set as:

$$
\omega=\omega_{\max }-\frac{t \times\left(\omega_{\max }-\omega_{\min }\right)}{t_{\max }}
$$

Where, $t_{\max }$ is the total iteration number; $\omega_{\max }$ and $\omega_{\min }$ are maximum and minimum inertia factors respectively.

Based on the distance between the particles and global optimum value, $\omega$ will be adjusted as the following:

$$
\omega=\omega_{0} \times\left(1-\frac{\text { dist }_{i}}{\text { dist }_{\max }}\right)
$$

Where, $\omega_{0}=\operatorname{rand}(0.5,1)$, and dist $_{i}$ is the Euclidean distance between the current particle and global optimum particle.

$$
\operatorname{dist}_{i}=\left[\sum_{j=1}^{n}\left(p_{g j}-x_{i j}\right)^{2}\right]^{\frac{1}{2}}
$$

dist $_{\max }$ is the maximum of dist $_{i}$.

This adjustment for $\omega$ can provide the particle does not deviate too far from the optimal value due to the gravity between the particle value and global optimal value when the particle is deviated the global optimal particle.

\section{Experimental Analysis}

Swarm size dimension: $N=10, D=2$; the maximum number of iterations of $T_{\max }=50 ;$ inertia weight $\omega_{\max }=0.9$ and $\omega_{\min }=0.4$, learning factor $C_{1}=1.4324, C_{2}=1.59612$; Gauss mutation probability $p_{m}=0.5$. Experimental environment for running: Intel Xeon E5-2620, CPU 2.5GHz, and Matlab2010b. Image segmentation results generated by the new algorithm are compared with that generated by the standard PSO algorithm.

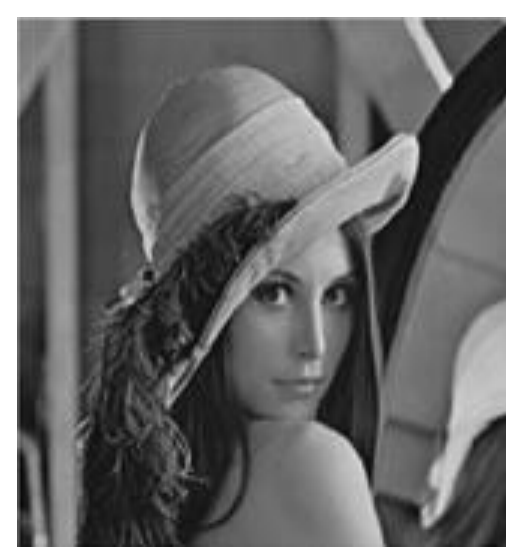

(a) Original Image 

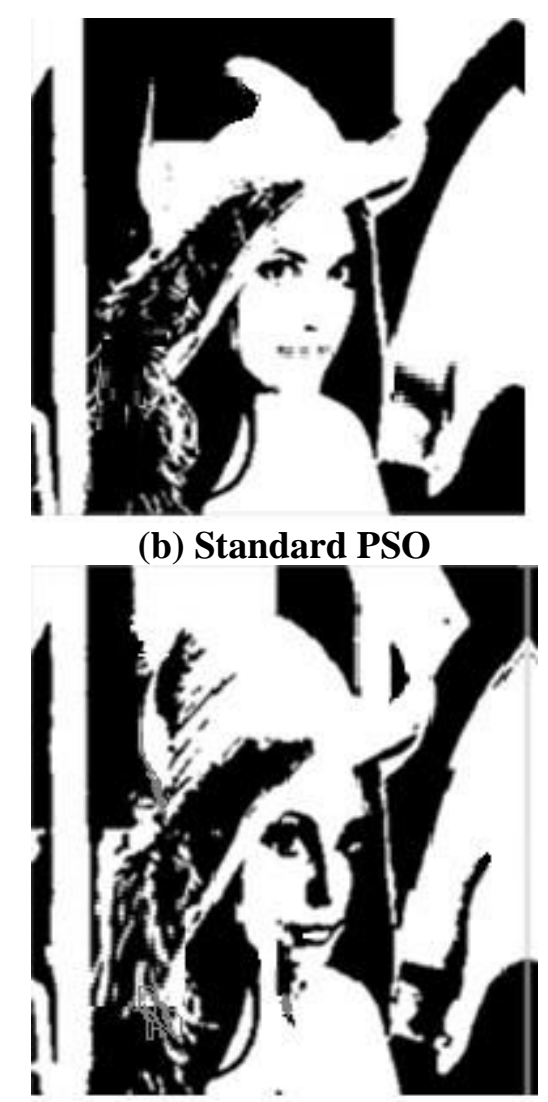

(c) The New Algorithm

Figure 3. Comparison of Different Algorithms

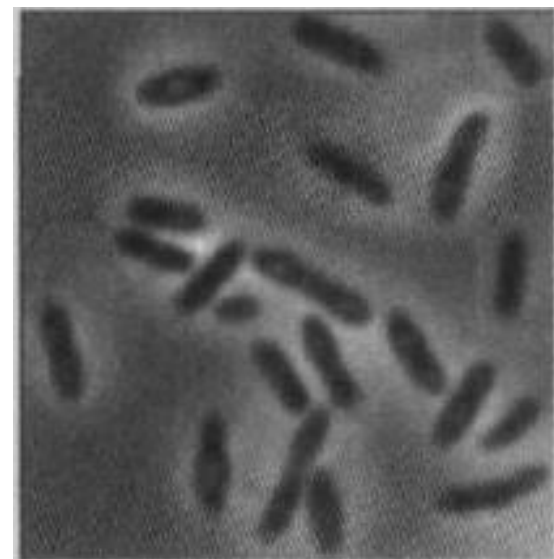

(a) Original Image 


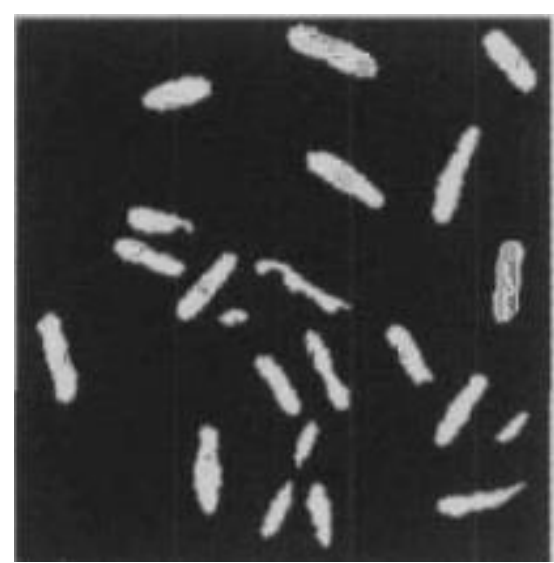

(b) Standard PSO

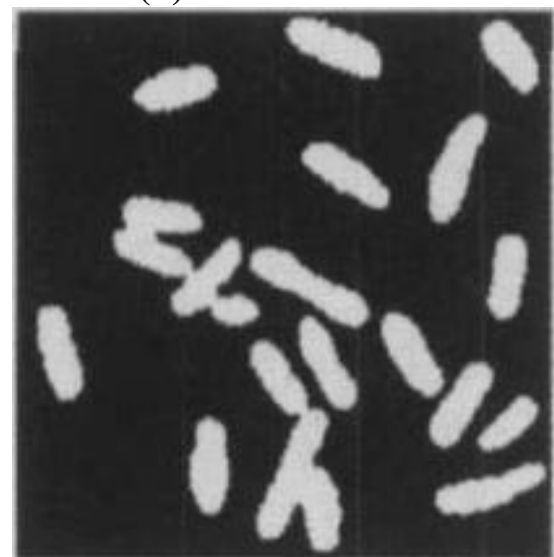

(c) The New Algorithm

\section{Figure 4. Comparison of Different Algorithms}

From the Figure 3 and Figure 4, we can see the new algorithm is effective. Digital media image segmentation by the new algorithm can get better effect.

\section{Conclusions}

Continuous development of computer science and technology provides the working environment for the digital media image processing. New treatment methods have been proposed by many researchers. The digital image is effective for various researches. In analysis, image segmentation can be seen as a particular region to separate from the other parts of the image. That means that the digital media image segmentation divides the image into a number of regions with specific and unique natures. It is the key step for the image processing.

In the paper, we propose a new algorithm for digital media image segmentation. The algorithm is based on asynchronous particle swarm optimization algorithm to obtain the adaptive threshold and take the inertia factor into the algorithm to get the optimal threshold for the image segmentation.

According to the experimental test, the method is verified effective for the digital media image segmentation. 


\section{References}

[1]. K. Parvati, B. S. P. Rao and D. M. Mariya, "Image segmentation using gray scale morphology and marker- controlled watershed transformation", Discrete Dynamics in Nature and Society, (2008), pp. 1- 8.

[2]. J. Serra, "Image Analysis and Mathematical Morphology", London, UK, Academic Press, (1982).

[3]. O. R. Victor, I. G. L. Juan, S. L. Nicolas and G. V. Pedro, "An improved watershed algorithm based on efficient computation of shortest paths", Pattern Recognition, vol. 40, no. 3, (2007), pp. 1078- 1090.

[4]. J. Serra, "Mathematical Morphology", London, UK, Academic Press, (1982).

[5]. M. G. Masoo leh and S. A. S. Moosavi, "An improved fuzzy algorithm for image segmentation", Proceedings of World Academy of Science, Engineering and Technology, vol. 4, (2008), pp. 400-404.

[6]. J. C. Bezdek James, J. Keller and K. Raghu, et al., "Fuzzy Models and Algorithms for Pattern Recognition and Image Processing”, Boston, Kluwer Academic Publisher, (1999).

[7]. Y. Wang, D. Liang and Y. Wang, "Transition region extraction and segmentation based on image fuzzy entropy neighborhood unhomogeneity", Acta Electronica Sinica, vol. 36, no. 12, (2008), pp. 2245- 2249.

[8]. B. Otman, Z. Hongwei and K. Fakhri, "Fuzzy Based Image Segmentation", Berlin: Springer-Verlag. (2003).

[9]. D. L. Wang and D. Terman, "Image segmentation based on oscillatory correlation", Neural Computation, vol. 9, (1997), pp. 805- 836

[10].S. Rout, S. Srivastava and J. Majumdar, "Multi modal image segmentation using a modified Hopfield neural network", Pattern Recognition, vol. 31, no. 6, (1998), pp. 743- 750.

[11].D. L. Vilarino, V. M. Brea and D. Cabello, 'Discrete Time CNN for Image segmentation by active contours", Pattern Recognition Letters, vol. 19, no. 8, (1998), pp. 721- 734.

[12].G. Kuntimad and H. S. Ranganath, "Perfect image segmentation using pulse coupled neural networks", IEEE Transactions on Neural Networks, vol. 10, no. 3, (1999), pp. 591- 598.

[13]. D. Dekruger and B. R. Hunt, "Image processing and neural networks for recognition of cartographic area feature", Pattern Recognition, vol. 27, no. 4, (1994), pp. 461- 483.

[14]. M. Goktepe, N. Yaiabik and V. Atalay, "Unsupervised segmentation of gray level Marko v model textures with hierarchical self-organizing map", Proceedings of the 13th International Conference on Pattern Recognition. USA: Institute of Electric and Electronic Engineer, (1996), pp. 90- 94.

[15]. Y. Wang, T. Adaii and S. Y. Kung, "Quantification and segmentation of brain tissues from MR images a probabilistic neural networks approach", IEEE Transactions on Image Processing, vol. 7, no. 8, (1998), pp. 1- 12.

[16].H. Berg, R. Olsson and T. Lindblad, "Automatic design of pulse coupled neurons for image segmentation", Neurocomputing, vol. 71, no. 6, (2008), pp. 1980- 1993.

[17].J, Zhang, X. Fan, J. Dong and M. Shi, "Image segmentation based on modified pulse- coupled neural networks", Chinese Journal of Electronics, vol. 16, no. 1, (2007), pp. 119- 122.

[18].L. Ting ,X. -b. Wen, J. -j. Quan, et al., "Multiscale SAR image segmentation using support vector machines", Proceeding s of the 2008 Congress on Image and Signal Processing, USA: IEEE, (2008), pp. 706- 709.

[19].O. Chapelle, P. Haffner and V. N. Vapnik, "Support vector machines for histogram- based image classification",IEEE Transactions on Neural Networks, vol. 10, no. 5, (1999), pp. 1055- 1064.

[20].V. N. Vapnik, "The Nature of Statistical Learning Theory", New York: Springer-Verlag, (2000).

[21].G. A. Bilodeau, et al., "Computerized medical imaging and graphics", Computerized Medical Imaging and Graphics, vol. 30, no. 7, (2006), pp. 437- 446.

[22].M. Pavan and M. Pelillo, "A new graph- theoretic approach to clustering and segmentation", Proc IEEE Conf Computer Vision and Pattern Recognition: USA: IEEE, (2003), pp. 145 - 152.

[23].W. Huang and L. Jiao, "Artificial immune kernel clustering network for unsupervised image segmentation", Progress in Natural Science, vol. 18, no. 4, (2008), pp. 455- 461.

[24].S. K. Pal and P. Mitra, "Multispectral image segmentation using the rough- se-t initialized EM algorithm", IEEE Transactions on Geoscience and Remote Sensing, vol. 40, no. 11, (2002), pp. 24952501 .

[25].L. A. Zadeh, "Fuzzy logic computing with words", IEEE Transactions on Fuzzy Systems, vol. 4, no. 2, (1996), pp. 103- 111.

[26].Z. Shi, Z. Zheng and Z. Meng, "Image segmentation-oriented tolerance granular space model", IEEE International Conference on Granular Computing, vol. 8, (2008), pp. 566-571.

[27].Y. Y. Yao, "Granular computing: basic issues and possible solutions", Atlantic: Association for Intelligent Machinery Press, (2000). 


\section{Authors}

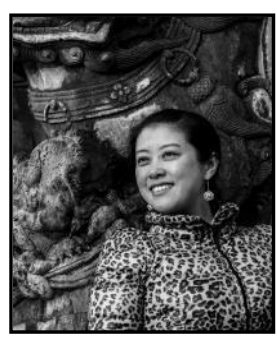

Wenchao Zhang, Graduated from North East Forestry University in 2011, Ph.D. Now work as a lecturer in the department of Art and Design, Changzhou College of Information Technology. The main research area is art and design.

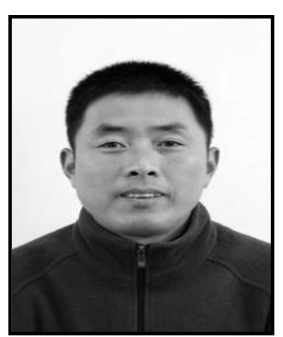

Caigen Zhou, Graduated from Shanghai Jiao Tong University in 2008, major in Administrative Management, Bachelor, Senior Engineer. Now work as the head of the department of Art and Design, Changzhou College of Information Technology. The main research area is electromechanical technology, cultural and creative industries.

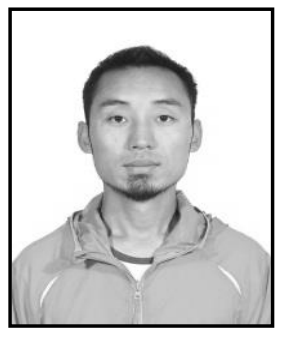

Xinxin Bao, Graduated from Beihua University in 2007, Bachelor. Now work as a teacher in the department of Art and Design, Changzhou College of Information Technology. The main research area is art and design. 